\title{
The Effect of Moderate-intensity Technical Training and Resistance Training on Selected Hematological Parameters of Elite Wrestlers
}

\author{
Serhat Özbay1,", Süleyman Ulupinar² \\ ${ }^{1}$ Faculty of Sport Science, Erzurum Technical University, Turkey \\ ${ }^{2}$ School of Health Sciences, Hacettepe University, Turkey
}

Copyright $\mathrm{O} 2018$ by authors, all rights reserved. Authors agree that this article remains permanently open access under the terms of the Creative Commons Attribution License 4.0 International License

\begin{abstract}
The aim of this study was to investigate the effect of an eight-week moderate-intensity technical and resistance training on selected hematological parameters. Twenty male wrestlers participated in the study voluntarily. Participants trained six days a week after being divided into technical and resistance training groups. The first group was applied a technical training (TT) program, at $60-75 \%$ intensity of heart rate (HR) and lasting 75-90 minutes. The other group (resistance training) applied the resistance training (RT) program for the first four weeks at $\% 60$ of 1TM load, for the next four weeks at $\% 70$ of 1TM load. Blood samples were collected from participants before the training period and after 24 hours following the last training session. RBC, HGB, HCT, PLT values were analyzed to determine hematological parameters from blood samples. Statistical analysis was performed using the SPSS program. According to the results, between values before and after the training period, there was significant difference in RBC and HGB levels, while there was no significant difference in HCT and PLT levels in the TT group. Between values before and after the training period, there was no significant difference in RBC, HGB, HCT, PLT levels in RT group. As a result, it was found that eight-week technical training was more efficient significantly than resistance training $(\mathrm{p}<0.05)$. However, when these results were examined in practical terms, it was determined that the differences between before and after technical training programs had a low effect size.
\end{abstract}

Keywords Technical Training, Resistance Training, Hematological Parameters

\section{Introduction}

Exercise is considered as a stressing factor on metabolism (1-4). The physiological adaptation of athletes exposed to continuous exercise is one of the scientific interesting points for researchers (5-7). In response to exercise, some metabolic and physiological changes may occur as acute or chronic. Enzyme activity, hormones and changes in cell numbers are the parameters obtained through blood to examine the responses to exercise (8-10). Exercise is known to affect some blood parameters as acute due to stress (10-12). On the other hand exercise may cause some chronic effects if repeated regularly $(10,13,14)$. These effects are influenced by many factors such as the type, intensity and duration of the exercise $(4,15,16)$.

Researchers emphasize that low-to-moderate regular aerobic exercise for healthy life is useful in balancing blood parameters $(9,17)$. However, there are different the opinions about enzyme activities and hematological values of athletes. Some researchers have found that blood parameters continue to be within normal limits in athletes trained regularly $(1,3,17)$, while some of them have put forth that these values may be outside the normal range (18, 19).

One of the methods commonly used to determine the effects of exercise-induced stress on the body is to evaluate the data obtained from blood samples. Measuring the concentration of blood cells to determine the effect of exercise is method mostly used in scientific research $(2,5)$. RBC, WBC, HGB, HCT and PLT values are the most investigated hematological variables related to exercise $(5$, $8,17)$.

Changes in blood parameters depending on the intensity of exercise have been examined in many studies $(11,12$, 20). Generally, while the researches have examined the effects of exercise on blood parameters as acute $(18,19)$, the studies that have chronically examined only concentrate on one type of training program $(11,21)$. However, in cases which the intensity of exercise is within similar, the studies on whether blood parameters are 
affected by the exercise type is quite limited. Therefore in this study, we aimed to investigate the chronic effects of two different moderate-intensity training protocols on selected hematological parameters.

\section{Method}

This study was a randomized experimental study without control group. Two groups with similar characteristics were exposed to different training programs during eight weeks and the changes in hematological parameters were examined.

\section{Participants}

Twenty male Greco-Roman wrestlers (age: $19.6 \pm 1.6$ years; height: $168.1 \pm 9.1 \mathrm{~cm}$; body mass: $65.7 \pm 7.9 \mathrm{~kg}$ ) were participated in the study. Participants were randomly divided into technical training (TT) and resistance training (RT) groups. Participants consisted of individuals who regularly train for at least three years (5-9 times a week) and vie in national competitions. Persons with temporary injury or illness were excluded from the study. Participants volunteered to participate in this study and signed informed consent.

\section{Training Programs}

Both training groups performed total 48 training sessions, during eight weeks, six days a week. In a training session, TT group performed 15-20 min warming up and gymnastics, 45-60 min technique, 5 min stretching exercise In this sessions, 10 different techniques were performed in three sets which lasted one minute. Participants tried to reach the maximum number of repetitions in sets. After the set was completed, participants were rested until their heart rate (HR) reduce the $\% 60$ of HRmax (220-age). Heart rate was measured during rest using a heart rate monitor (S610i, Polar Electro Oy, Finland). The RT group performed six different resistance exercises consisting of lower and upper body exercises. These exercises were applied in three sets. Three minute rest periods were used between exercises and sets. Before starting the study, 1RM loads were determined for each exercise. Then, they performed a maximum number of repetitions in $60 \%$ of $1 \mathrm{RM}$ loads in the first four weeks, and in $70 \%$ of $1 \mathrm{RM}$ loads in the second four weeks.

\section{Statistical analysis}

For all statistical analyses, SPSS program was used. Data were analyzed by using mean and standard deviation values. The normality of the data was detected with the Shapiro-Wilk test. Homogeneity of variances were detected with Levene's test. The paired sample t-test was used to determine the effect of training methods on the variable (before-after). To determine the practical significance of the differences, the effect size was calculated according to the Cohen's d formula (22). Effect size was assessed as low (0.2), medium (0.5) and high (0.8). Significance level was determined as $<0.05$ for all statistical processing.

\section{Results}

It was found that there was no difference between the groups in descriptive characteristics and hematological parameters (Table 1).

Table 1. Descriptive characteristics and hematological values of the groups at the beginning.

\begin{tabular}{ccccc}
\hline & $\mathrm{TT}$ & $\mathrm{RT}$ & $\mathrm{t}$ & $\mathrm{p}$ \\
\hline Age $($ years $)$ & $20.40 \pm$ & $19.30 \pm 1.41$ & 1.601 & 0.127 \\
& 1.64 & & & \\
Height $(\mathrm{cm})$ & $168.20 \pm$ & $169.90 \pm 6.15$ & 0.529 & 0.603 \\
& 8.08 & & & \\
BM $(\mathrm{kg})$ & $66.46 \pm$ & $64.86 \pm 5.03$ & 0.426 & 0.675 \\
& 10.74 & & & \\
$\mathrm{RBC}\left(10^{3} / \mu \mathrm{l}\right)$ & $5.27 \pm$ & $5.08 \pm 0.37$ & 0.998 & 0.331 \\
& 0.46 & & & \\
$\mathrm{HGB}(\mathrm{g} / \mathrm{dl})$ & $14.35 \pm$ & $14.36 \pm 1.16$ & 0.011 & 0.991 \\
& 1.28 & & & \\
$\mathrm{HCT}(\%)$ & $38.98 \pm$ & $35.70 \pm 12.43$ & 0.799 & 0.434 \\
& 3.71 & & & \\
$\mathrm{PLT}\left(10^{3} / \mu \mathrm{l}\right)$ & $284.30 \pm$ & $270.40 \pm 39.40$ & 0.871 & 0.395 \\
\hline
\end{tabular}

TT: Technical training group, RT: Resistance training group

RBC: Erythrocyte, HGB: Hemoglobin, HCT: Hematocrit, PLT: Platelet, BM: Body mass

Hematological parameters of TT group before and after training were examined (Table 2). According to the results, there was significant decrease in RBC and HGB values ( $p$ $<0.05)$. When the effect size of these results were examined, it was found that RBC (0.40) and HGB (0.36) variables had a low effect size. Besides, there was no significant difference in HCT and PLT values.

Table 2. Change in the Hematological Parameters of Technical Training Group $(\mathrm{n}=10)$

\begin{tabular}{cccccc}
\hline & Before & After & $\mathrm{t}$ & $\mathrm{p}$ & $\mathrm{ES}$ \\
\hline $\mathrm{RBC}$ & $5.27 \pm$ & $5.11 \pm 0.35$ & 2.753 & $0.022^{*}$ & 0.40 \\
$(103 / \mu \mathrm{l})$ & 0.46 & & & & \\
$\mathrm{HGB}(\mathrm{g} / \mathrm{dl})$ & $14.35 \pm$ & $13.94 \pm 1.00$ & 2.941 & $0.016^{*}$ & 0.36 \\
& 1.28 & & & & \\
HCT $(\%)$ & $38.98 \pm$ & $39.76 \pm 3.26$ & -1.82 & 0.102 & \\
PLT & 3.71 & & 2 & & \\
$(103 / \mu \mathrm{l})$ & 31.51 & $279.80 \pm 55.61$ & 0.300 & 0.771 & \\
\hline
\end{tabular}

* There is a significant difference the values between before and after $(\mathrm{p}<0.05)$.

RBC: Erythrocyte, HGB: Hemoglobin, HCT: Hematocrit, PLT: Platelet, ES: Effect size

Hematological parameters of the RT group before and after the exercise were examined (Table 3 ) and there was no significant difference in RBC, HGB, HCT and PLT values. 
Table 3. Change in Hematological Parameters of Resistance Training Group $(\mathrm{n}=10)$

\begin{tabular}{ccccc}
\hline \multicolumn{1}{c}{ Before } & After & $\mathrm{t}$ & $\mathrm{p}$ \\
\hline $\mathrm{RBC}$ & $5.08 \pm 0.37$ & $5.15 \pm 0.28$ & -0.896 & 0.394 \\
$\left(10^{3} / \mu \mathrm{l}\right)$ & & $14.50 \pm$ & & \\
$\mathrm{HGB}$ & $14.36 \pm 1.16$ & $\begin{array}{c}0.97 \\
\mathrm{~g} / \mathrm{dl})\end{array}$ & -0.846 & 0.420 \\
$\mathrm{HCT}(\%)$ & $35.70 \pm 12.43$ & $\begin{array}{c}40.99 \pm \\
2.52\end{array}$ & -1.260 & 0.239 \\
$\mathrm{PLT}$ & & $266.40 \pm$ & 0.606 & 0.560 \\
$\left(10^{3} / \mu \mathrm{l}\right)$ & $270.40 \pm 39.40$ & 38.56 & & \\
\hline
\end{tabular}

* There is a significant difference the values between before and after $(\mathrm{p}<0.05)$.

RBC: Erythrocyte, HGB: Hemoglobin, HCT: Hematocrit, PLT: Platelet

\section{Discussion and Conclusion}

In this study, effect of technical and resistance training program, which have the same period (eight weeks) and intensity (moderate intensity), on some hematological parameters were investigated. In TT group, between before and after training period, there was significant decrease in RBC and HGB values was found. There was no significant difference in HCT and PLT values. In RT group, between before and after training period, there was no significant difference in RBC, HGB, HCT and PLT values. However, when the effect size of these results were examined, it was determined that the effect size was low (Cohen, 1988) in the differences obtained in TT group.

Effect of exercise on hematological parameters are one of the methods most researched by researchers $(2,12,23)$. For example, in a study, the participants completed the half marathon $(21.1 \mathrm{~km})$ run to determine the effect of exercise on some hematological parameters. Blood samples were collected before running, just after running, after 3 hours and after 20 hours. According to the results of the study, RBC and HGB values decreased significantly following half marathon running. It was also found that the $\mathrm{RBC}$ and HGB values after 3 hours and 20 hours were significantly lower than the values before running. It was found that after running PLT values are significantly higher than the values before the running. However there was no significant difference between before running, after 3 hours and after 20 hours (2). In another study, changes in hematological values after aerobic and anaerobic exercise were examined. As a result of the study, no significant changes in all hematological parameters were found after aerobic exercise. However, there was a significant increase in HGB, HCT, WBC values just after anaerobic exercise, while there was a significant decrease after 24 hours. When the results of both exercises were compared, it was found that the increases and decreases in anaerobic exercise were significantly higher than the aerobic exercise (12). In these studies the acute effect of exercise were examined on hematological parameters, whereas in our study the chronic effect of an eight-week training period was examined.

The chronic effect of exercise on hematologic parameters has been examined in different ways in studies $(11,13,23)$. For example, in a study, HGB, HCT, PLT, WBC and RBC values of taekwondo athletes were examined before and after the national team camp. At the end of the study, it was found that the period of four weeks training did not cause a significant change in HGB, HCT, PLT and WBC values, but RBC values were significantly increased. This increase in $\mathrm{RBC}$ values is emphasized to be low level (11). This study shows similar results with our research in terms of the fact that the training partially affects hematological values and this effect is low. In another study that lasted seven weeks, intensive and extensive interval training program was applied. At the end of the study, there was a significant increase in RBC, HCT and HGB levels in the extensive interval program group, whereas in the intensive interval program group only PLT levels decreased significantly (13). In another study, the effect of sea level and high altitude aerobic exercises on some hematological parameters were investigated. The participants had aerobic training at high altitude for 15 days after sea level blood samples were collected and they were returned to sea level again. At the end of the study, after 15 days of high altitude training, significant increase in WBC and HGB values was found. There was no significant change in RBC and HCT values. Eight days after returning to sea level WBC values were significantly decreased, but no significant change was found in HGB, RBC and HCT values (23). In another study applied on mice, no significant difference was found between exhaustive swimming exercise group and control group in terms of RBC, HGB and HCT during four-weeks. However, it was found that PLT values decreased significantly in exercise group (16).

Although the results of our study have been supported by many researchers in the literature $(1,3,4,23)$, there are also studies which different results have been reached $(2$, 12). In general, it was observed that the training periods such as 4-12 weeks did not cause great changes on hematological parameters $(1,3,4,11,23)$. In our study, although there was statistically significant difference in terms of some hematological parameters between before and after the eight-week technical or resistance training program, it was found that the practical effect of these differences was low.

As a result, in this study, it has been found that different types of exercise applied with moderate severity have a statistically significant effect on some hematological parameters. However, when these results were evaluated in terms of practical significance, the effect size of all the differences was found to be low.

\section{REFERENCES}

[1] Houghton D, Hallsworth K, Thoma C, Cassidy S, Hardy T, Heaps $\mathrm{S}$, et al. Effects of exercise on liver fat and 
metabolism in alcohol drinkers. Clinical Gastroenterology and Hepatology. 2017; 15(10):1596-603. e3.

[2] Lippi G, Salvagno GL, Danese E, Tarperi C, Guidi GC, Schena F. Variation of red blood cell distribution width and mean platelet volume after moderate endurance exercise. Advances in hematology. 2014; 2014.

[3] Tartibian B, Khayat SMA, Asgarzadeh R. Transforming Growth Factor- $\alpha$ Gene Expression and AST, ALT and Lipase Serum Levels Changes in Response to 8 Weeks Resistance Training. Iranian Journal of Allergy, Asthma \& Immunology. 2018;17.

[4] Yao J, Meng M, Yang S, Li F, Anderson RM, Liu C, et al. Effect of aerobic and resistance exercise on liver enzyme and blood lipids in Chinese patients with nonalcoholic fatty liver disease: a randomized controlled trial. Int J Clin Exp Med. 2018; 11(5):4867-74.

[5] Cinar V, Mogulkoc R, Baltaci AK. Calcium supplementation and 4-week exercise on blood parameters of athletes at rest and exhaustion. Biological trace element research. 2010; 134(2):130-5.

[6] Peake JM, Neubauer O, Walsh NP, Simpson RJ. Recovery of the immune system after exercise. Journal of Applied Physiology. 2016;122(5):1077-87.

[7] Simpson RJ, Kunz H, Agha N, Graff R. Exercise and the regulation of immune functions. Progress in molecular biology and translational science. 135: Elsevier; 2015. p. 355-80.

[8] Neufer PD, Bamman MM, Muoio DM, Bouchard C, Cooper DM, Goodpaster $\mathrm{BH}$, et al. Understanding the cellular and molecular mechanisms of physical activity-induced health benefits. Cell metabolism. 2015;22(1):4-11.

[9] Şenişik SÇ. Exercise and the immune system. Turkish Journal of Sports Medicine. 2015;50(1):011-20.

[10] Soslu R, Eyüboğlu E, Çuvalcioğlu İC, Özkan A. Effects of Blood Parameters and Some Morphological Variables on Some Upper Extremity Physical Fitness Performance in Female Basketball Players. İnternational journal of cultural and social studies(IntJCSS). 2017; 3(Special Issue 2):347-53

[11] Çakmakçı E. Effects of camp term on some hematological parameters in male taekwondoers. Niğde University Journal of Physical Education and Sports Sciences. 2009.

[12] İbiş S, Hazar S, Gökdemir K. Acute effect of hematological parameters on aerobic and anaerobic exercise. Journal of
Human Sciences. 2010;7(1):71-81.

[13] Demiriz M. Comparison of the Effects of Anaerobic Interval Training with Different Rest

Interval on Aerobic Capacity, Anaerobic Threshold and Blood Parameters. Master Thesis. Balıkesir University. Institute of Health Sciences. 2013.

[14] Kaynar Ö. The Effect of Training on Pituitary Gland Hormones and Liver Enzymes in Elite Wrestlers. Master Thesis. Atatürk University. Graduate School of Health Sciences. 2014

[15] Turner JE. Is immunosenescence influenced by our lifetime "dose" of exercise? Biogerontology. 2016;17(3):581-602.

[16] Yuan X, Xu S, Huang H, Liang J, Wu Y, Li C, et al Influence of excessive exercise on immunity, metabolism, and gut microbial diversity in an overtraining mice model. Scandinavian journal of medicine \& science in sports. 2018;28(5):1541-51.

[17] Kara E, Özal M, Yavuz HU. Comparison of the blood parameters and respiratory functions of elite wrestlers and basketball players. Selçuk University Journal Of Physical Education And Sport Science. 2010; 12(1):36-41.

[18] Kaynar Ö, Öztürk N, Kiyici F, Baygutalp NK, Bakan E. The Effects of Short-Term Intensive Exercise on Levels of Liver Enzymes and Serum Lipids in Kick Boxing Athletes. Dicle Medical Journal. 2016;43(1):130.

[19] Shamsoddini A, Sobhani V, Chehreh MEG, Alavian SM, Zaree A. Effect of aerobic and resistance exercise training on liver enzymes and hepatic fat in Iranian men with nonalcoholic fatty liver disease. Hepatitis monthly. 2015;15(10).

[20] Turgut M, Çınar V, Pala R, Karaman ME. Effects of acute exercise on some biochemical parameters of women. European Journal of Physical Education and Sport Science, 2017.

[21] Akbulut E. The Effects of Aerobic Exercises on Body Composition and Blood Lipids in Sedentary Women. Master Thesis. Selçuk University. Graduate School Of Health Sciences, 2011.

[22] Cohen J. Statistical power analysis for the behavioral sciences. 2nd. Hillsdale, NJ: erlbaum; 1988.

[23] Kaya I. The effect of extensive interval training at altitude on the physiological, aerobic, anaerobic and various blood parameters of athletes. SHS Web of Conferences; 2016: EDP Sciences. 